\title{
Vaccine inequality benefits no one
}

\author{
It is seemingly in the interest of high-income countries to prioritize vaccinating their own population against \\ COVID-19, despite it being immoral. However, mathematical modelling by Ye et al.' shows that this approach offers \\ only limited, short-term benefits, whereas equitable vaccine distribution would substantially curb the emergence \\ and spread of new variants.
}

\section{Dan Yamin}

T he year 2020 witnessed a game-changing historical moment - the COVID-19-driven vaccine revolution. Within less than 11 months, the first vaccine was approved by regulatory agencies. High-income countries (HICs) responded immediately by entering into bilateral deals with vaccine suppliers, racing to reach herd immunity, which was later augmented by booster-shot programmes.

With the stated vision 'no one is safe, unless everyone is safe', the global COVAX Facility initiative was established to coordinate international resources to ensure low- and middle-income countries (LMICs) have equitable access to COVID-19 vaccines. Working with governments and manufacturers, and backed and funded by the World Health Organization (WHO) and many HICs, nearly a billion vaccine doses have been shipped by COVAX to 144 countries so far ${ }^{2}$. Despite these efforts, there remains a stark and growing imbalance in COVID-19 vaccine coverage worldwide. For example, low-income countries account for $8 \%$ of the world population, but have secured less than $1 \%$ of the total vaccine doses distributed around the globe to date ${ }^{3}$.

A novel mathematical model published in Nature Human Behaviour by Ye et al. ${ }^{1}$ reveals that this self-prioritization strategy of HICs might facilitate the emergence of new variants of concern. The model integrates multistrain metapopulation information with air-traffic data to capture transmission routes within and between countries. The authors evaluated the effectiveness of vaccine distribution strategies in reducing COVID-19 cases and deaths. Specifically, they examined the effectiveness of vaccine distribution strategies on the basis of multiple prioritization factors, including countries' population size and ongoing morbidity or mortality rates, in equitable and inequitable settings.

The modelling results suggest that vaccine inequity provides only limited

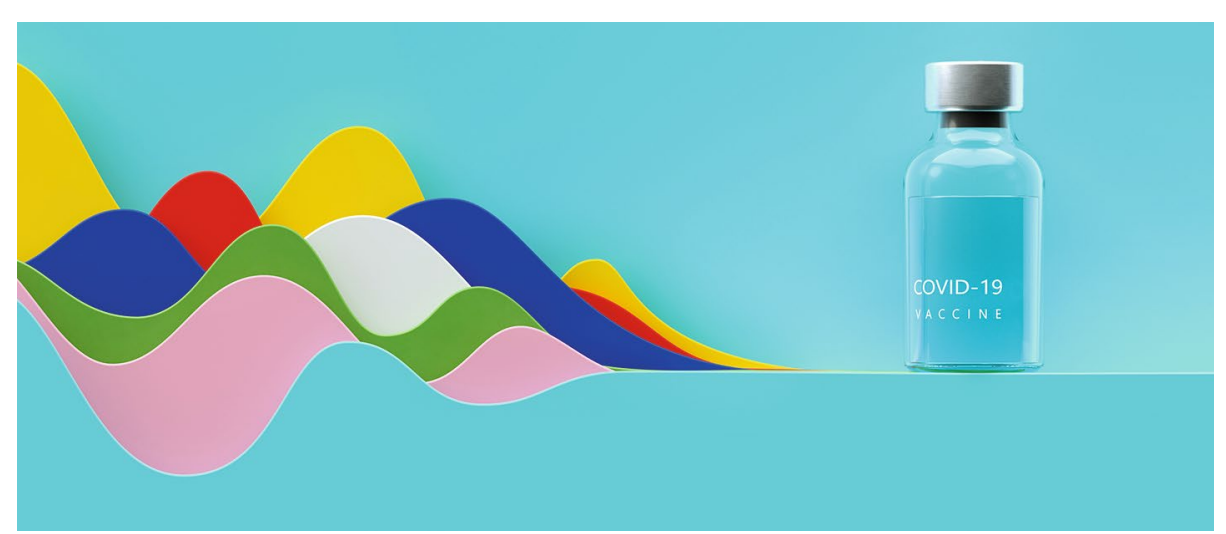

Credit: Andriy Onufriyenko / Moment / Getty

and short-term benefits to HICs, but is detrimental in the long term. Conversely, allocating the pool of vaccines equitably among sovereign states would substantially curb the emergence and spread of new variants in all countries. Another finding in favour of equitable distribution is that a sharper disparity in vaccine allocation between high- and low-income countries would inevitably shorten the time lag between waves and increase the number of infected individuals in each wave.

Although mutations occur by chance, multiple compounding factors of disadvantage may facilitate the spread of variants in low-resource settings. Specifically, high population density and large household size are known to exacerbate COVID-19 transmission ${ }^{4}$ and, consequently, the prevalence of mutations. Other potentially contributing factors include hygiene and wastewater treatment infrastructure, as well as limited outbreak management capacity ${ }^{5}$. Moreover, a larger fraction of the population in low-income settings is immunocompromised. Multiple studies have shown that immunocompromised individuals exhibit persistent viral shedding of COVID-19 (ref. ${ }^{6}$ ). This extended duration of infection affords the coronavirus more time to evolve the means to evade the immune response, the potential outcome of which is a new, more immune-resistant strain.

As a practical approach, the authors suggest that if HICs donate a certain portion of their vaccine supplies to LMICs instead of vaccinating their entire population as the top priority, enormous public health benefits would be reaped by both HICs and LMICs. Although indirect benefits from protecting neighbouring LMICs has been demonstrated for other epidemics, such as seasonal influenza ${ }^{7}$, the authors point out that in the case of the COVID-19 pandemic this is not the most beneficial strategy, and that a global approach is required.

A potential limitation of their study is that viral evolutionary dynamics was modelled in a simple linear form, in which the severity of new strains always increases. However, selection against severe strains may happen as the virus evolves. On that note, only time will tell whether the highly transmissible, but less virulent, Omicron variant will boost the immunity of recovered individuals, thereby protecting them against future virulent variants. Expanding their 
model to include the selection of strains is an important topic for further exploration when more data about different strains become available.

Far greater and more long-lasting protection against COVID-19 can be obtained through global cooperation. A large-scale survey conducted in late 2020 in 7 HICs suggested that over $70 \%$ of their population support prepurchased vaccine donations to any low-income countries. Strong grassroots support is a key factor in shaping governments' willingness to execute this approach and subsidize vaccines in low-income countries ${ }^{8}$. In summary, subsidizing COVID-19 vaccination in LMICs is a practically sound, moral obligation of HICs - and it also happens to be in their own self-interest.

\section{Dan Yamin (iD) $1,2 \bowtie$}

${ }^{1}$ Laboratory for Epidemic Modeling and Analysis, Department of Industrial Engineering, Faculty of Engineering, Tel Aviv University, Tel Aviv, Israel. ${ }^{2}$ Center for Combatting Pandemics, Tel Aviv University, Tel Aviv, Israel.

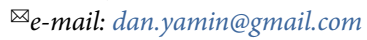

Published online: 31 January 2022 https://doi.org/10.1038/s41562-022-01297-8
References

1. Ye, Y. et al. Nat. Hum. Behav., https://doi.org/10.1038/s41562-02201289-8 (2022).

2. UNICEF Supply Division. COVID-19 vaccine market dashboard. unicef.org, https://go.nature.com/3A19TnF (accessed 17 January 2022).

3. WHO. WHO coronavirus (COVID-19) dashboard. covid19. who.int, https://covid19.who.int/info/ (accessed 17 January 2022).

4. Patel, J. A. et al. Public Health 183, 110-111 (2020).

5. Arslan, M., Xu, B. \& Gamal El-Din, M. Sci. Total Environ. 743, 140709 (2020).

6. Aydillo, T. et al. N. Engl. J. Med. 383, 2586-2588 (2020).

7. Yamin, D., Kahana, D., Shahmoon, E., Fitzpatrick, M. C. \& Galvani, A. P. BMC Public Health 21, 1543 (2021).

8. Clarke, P. M. et al. Nat. Med. 27, 935-936 (2021).

Competing interests

The author declares no competing interests. 